\title{
Homeostasis Processes Expressed as Flashes in a Poincaré Sections
}

\author{
Yehuda Roth \\ Oranim Academic College, Oranim Campus, K. Tivon Town, Israel \\ Email: yudroth@gmail.com
}

How to cite this paper: Roth, Y. (2018) Homeostasis Processes Expressed as Flashes in a Poincaré Sections. Journal of Modern Physics, 9, 2135-2140. https://doi.org/10.4236/jmp.2018.912134

Received: September 12, 2018

Accepted: October 8, 2018

Published: October 11, 2018

Copyright $\odot 2018$ by author and Scientific Research Publishing Inc. This work is licensed under the Creative Commons Attribution International License (CC BY 4.0).

http://creativecommons.org/licenses/by/4.0/

\section{cc) (i) Open Access}

\begin{abstract}
We describe a homeostasis system with a discrete map that is revealed by stroboscopic "flashes" (Poincaré sections) that are synchronized with the measurement events.
\end{abstract}

\section{Keywords}

Homeostasis, Poincaré Sections, Regulation, Discrete Map

\section{Introduction}

Homeostasis is a process that corresponds with biological measurements that are conducted over the internal and external body environments. Its purpose is to keep the body in a steady phase under a varying environment [1]. A regulation is obtained by a negative feedback process, as described in Figure 1. A negative feedback system has a sensor that monitors a physiological value. The collected data is transferred to the control center in the brain that compares the received data to the normal range. If the value is far from the set point, then the control center activates an effector which, through the negative feedback loop, balances the value to the normal range. For example, in a thermo-regulation process, sensors in the blood vessels are constantly sending the brain updates on internal temperatures. This information is sent to the hypothalamus area, where four different types of neurons analyze the data. Then, if necessary, an effector is triggered to balance the temperature to the range of the fixed point [2] (see also ref. [3] [4] for the Hammel's model and ref. [5] for an extensive review).

Oscillatory behavior in a range of $10-100[\mathrm{~ms}]$ is commonly observed in many neurons [6]. Because Homeostasis processes are activated by the brain, we propose that the time between these measurement events will be at that scale. Negative feedback systems are mostly described by recursive maps [7] [8]. By 


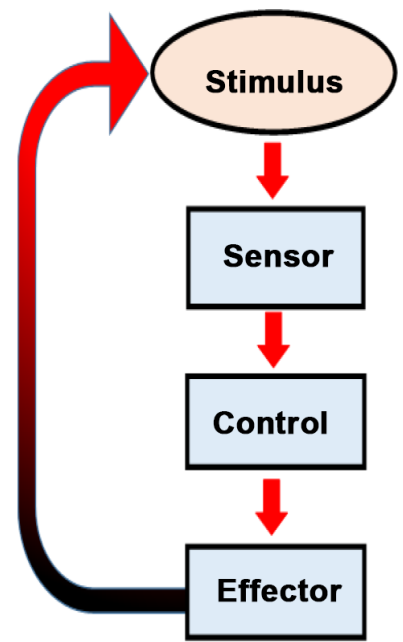

Figure 1. Feedback loop description: Data is collected by the sensors and transferred to the control center. If the value is far from the set point, then the effector, through the negative feedback loop, balances the value to the normal range.

synchronizing the maps' evolution with the measurement events, we describe a regulation process by the Poincaré stroboscopic "flashes". Note that although negative feedback corresponds with nonlinear maps' in practice, we can also analyze maps that in the transition from the $n$ to the $n+1$ stages are linear.

\section{Maps}

Suppose that $\sigma$ are a set of numbers over $\mathbb{R}$. The measurement's dynamics are determined by a discrete map $\mathbf{S}$ : in a Poincaré stroboscopic style where the "flashes" are synchronized with the neuron detections events. Once an initial value $\sigma_{0}$ is detected, the homeostasis iteration starts, such that

$$
\mathbf{S}: \sigma_{n}=\sigma_{n+1} .
$$

Considering a regular map that converges to a single value $\sigma_{\infty}$ such that

$$
\lim _{n \rightarrow \infty} \mathbf{S}: \sigma_{n}=\sigma_{\infty} .
$$

where $\sigma_{\infty}$ is the regulation point such as $37^{\circ} \mathrm{C}$ in the temperature regulation.

\section{Homeostasis Process Expressed by Linear Maps}

In this part we introduce the general description of Homeostasis maps. This will be demonstrated for temperature regulation process in the following subsection.

\section{General Description of Homeostasis Linear Maps}

The numerical values of $\sigma$ depend on the system-unit such as the Celsius or Fahrenheit scales for temperature detections. Consider two observers who implement different system units I and II-say, the Celsius and Fahrenheit scales-to measure the same process. Mathematically speaking, the conversion is 
obtained by the linear combination,

$$
\sigma[\mathrm{I}]=A \sigma[\mathrm{II}]+B \text {. }
$$

For example, converting from [Celsius] to [Fahrenheit] corresponds with,

$$
T\left[{ }^{\circ} \mathrm{F}\right]=1.8 T\left[{ }^{\circ} \mathrm{C}\right]+32 .
$$

At the initial stage, where $n=0$, both observers measure the same state but with different numerical values. Actually, they describe the same data. Our aim is to find a recursive relation that conserves the converting formula between different measurement units.

For any arbitrary functions $f_{1}$ and $f_{2}$, a recursion relation that conserves the conversion equation must be of the form:

$$
f_{1}\left(\frac{\sigma_{n+1}-\sigma_{1}}{\sigma_{3}}\right)=f_{2}\left(\frac{\sigma_{2}-\sigma_{n}}{\sigma_{4}}\right)
$$

where $\sigma_{1}, \sigma_{2}, \sigma_{3}$ and $\sigma_{4}$ are constants possessing the same units of measurement. A simple case is of the linear maps that appear as:

$$
\sigma_{n+1}-\sigma_{i}=a\left(\sigma_{e}-\sigma_{n}\right)
$$

where $\sigma_{i}$ is an internal reference parameter determined by the body's biology, such as the liver's desired temperature, $\sigma_{e}$ is an external parameter such as the external environment temperature and a stands for the rate of body-surrounding iteration. Simplifying this expression we obtain,

$$
\sigma_{n+1}=\sigma_{i}+a \sigma_{e}-a \sigma_{n}
$$

or

$$
\mathbf{S}: \sigma_{n}=\sigma_{i}+a \sigma_{e}-a \sigma_{n}
$$

This map fluctuates until it eventually reaches the final value $\sigma_{\infty}=\frac{\sigma_{i}+a \sigma_{e}}{1+a}$. The negative feedback can be observed if instead of expressing the transition $n \rightarrow n+1$ we follow iteration $n \rightarrow n+2$. Now, Equation (7) becomes

$$
\sigma_{n+2}=(1-a) \sigma_{i}+a(1-a) \sigma_{e}+a^{2} \sigma_{n}
$$

By comparing Equation (7) with Equation (9), we see that the parameters are rescaled such that $\sigma_{i} \rightarrow(1-a) \sigma_{i}$ and $\sigma_{e} \rightarrow(1-a) \sigma_{e}$ only now the negative term $-a \sigma_{n}$ is transformed into a positive variable $\rightarrow a^{2} \sigma_{n}$. This reflects the negative feedback process: while in the single iteration $\sigma_{n}$ reduces $\sigma_{n+1}$, the positive sign in the proceeding iteration increase $\sigma_{n+2}$, and so on. This fluctuation decays along the iterations until the map reaches a final value, as shown in Figure 2.

\subsection{Temperature Regulation Maps}

Core body temperature, $T_{c}$, refers to the temperature of the internal body environment, such as the liver temperature. Relatively to the other body zones, $T_{c}$ varies in a very small range [9], which makes it appropriate to serve as an 


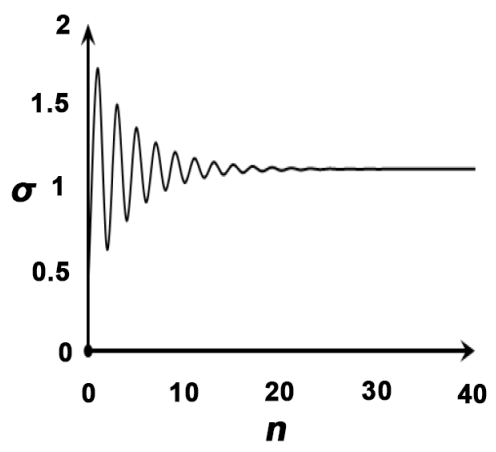

Figure 2. Graphs describing the map $\mathbf{S}: \sigma_{n}=\sigma_{r}-a \sigma_{n}$.

internal reference temperature $\left(\sigma_{i}=T_{i}\right)$. We also consider $\sigma_{e}=T_{s}$, where $T_{s}$ is the external environment temperature.

Suppose that at a stage $n$, the sensors detect a temperature of $T_{n}$ that is lower than $T_{c}$. This means that at that time the body emits heat calculated as $H_{\beta}=\beta\left(T_{n}-T_{s}\right)$, where $\beta$ represents a body isolation factor, such that, in the extreme scenario where $\beta=0$, no heat is transmitted to the external environment. Following this measurement result, the body responds (in the $n+1$ iteration) by generating internal heat to increase its temperature. This is represented with the heat term $H_{\alpha}=\alpha\left(T_{c}-T_{n+1}\right)$ where $\alpha$ is a parameter representing the heat production rate.

By comparing the two terms, $H_{\beta}=H_{\alpha}$, we obtain the recursive equation,

$$
T_{n+1}=T_{c}+a T_{s}-a T_{n},
$$

where $a=\frac{\beta}{\alpha}$, is a dimensional variable that determines the body-surrounding heat exchange. This equation is with agreement with Equation (8).

For a sufficient number of iterations, the temperature is regulated at $T_{\infty}$, which satisfies the relation

$$
T_{n+1}=T_{n} \stackrel{\text { def }}{=} T_{\infty},
$$

where

$$
T_{\infty}=\frac{T_{c}+a T_{s}}{1+a} .
$$

For example, for $T_{c}=37^{\circ} \mathrm{C}, T_{s}=25^{\circ} \mathrm{C}$ and $a=0.034483$ we find the final temperature at $T_{\infty}=36.6^{\circ} \mathrm{C}$.

\subsection{Control of Temperature Level}

There are extreme cases in which the conventional regulation process is not sufficient to balance the body parameter. For that scenarios, the brain initiate an additional process that we refer to a $\mathbf{S}_{R}$ : map.

A few scenarios affect temperature regulation, such as:

1) The internal "heat engine" increases or decreases heat production as reflected through $\alpha$ in the heat term $H_{\alpha}=\alpha\left(T_{0}-T_{n+1}\right)$. In an extreme heat 
production such as occurs in a physical activity like jogging, the body initiate an additional process such as sweating. This we describe by $\mathbf{S}_{R}$ ::

2) The external environment temperature drops or increases dramatically. This is mostly reflected though $T_{s}$. For a cold surrounding environment in which heat runs away rapidly from the body environment, the control is on the isolation factor $\beta$. A behavioral control can include wearing warm clothing, which will reduce $\beta$. A physiological alternative is to initiate a $\mathbf{S}_{R}$ process, such as shivering that causes the muscles to produce extra heat.

Let us demonstrate the scenario of a temperature reduction process: For high $T_{s}$, the body temperature may reach temperatures higher than $37^{\circ} \mathrm{C}$. For example, using the earlier parameters $T_{c}=37^{\circ} \mathrm{C}$ and $a=0.034483$ but with $T_{s}=40^{\circ} \mathrm{C}$, we obtain $T_{\infty}=37.1^{\circ} \mathrm{C}$ (see Equation (11)). In this scenario, the body initiates $\mathbf{S}_{R}$ : in the following way: after applying the standard map $\mathbf{S}_{S}$ : the outcome temperature $T_{n+1}$ initiates the second map. Using a linear model while implementing the unit of measurements conservation, we define $\mathbf{S}_{R}$ : through the following recursion equation:

$$
T_{n+2}=T_{n+1}-b T_{s}+b T_{c}
$$

where $b$ is a new coefficient of the heat exchange term. By using Equation (10), we obtain that,

$$
T_{n+2}=(1-b) T_{c}+(a-b) T_{s}-a T_{n}
$$

where for $T_{n+2}=T_{n} \stackrel{\text { def }}{=} T_{\infty}$ we obtain,

$$
T_{\infty}=(1-b) T_{\infty}^{(s)}
$$

where $T_{\infty}^{(s)}$ is the standard regulation temperature (only now we added the superscript $(s)$ ) as appeared in Equation (12) By reusing the data $T_{c}=37^{\circ} \mathrm{C}$, $a=0.034483$ and $T_{s}=40^{\circ} \mathrm{C}$, that leads to $T_{\infty}^{(s)}=37.1^{\circ} \mathrm{C}$, we can apply $b=0.01348$ to obtain $T_{\infty}=36.6$.

\section{Summary}

We introduced simple maps that describe homeostasis processes. Finding fundamental equations that describe the homeostasis processes allows us to simplify its description and may contribute a new perspective in analyzing these processes.

\section{Conflicts of Interest}

The author declares no conflicts of interest regarding the publication of this paper.

\section{References}

[1] Homeostasis. https://www.nature.com/subjects/homeostasis

[2] Morrison, S.F. and Nakamura, K. (2011) Frontiers in Bioscience (Landmark Ed), 16, 74-104. https://doi.org/10.2741/3677 
[3] Hammel, H.T. (1965) Neurons and Temperature Regulation. In: Yamamoto, W.S. and Brobeck, J.R., Eds., Physiological Controls and Regulations, Saunders, Philadelphia, 71.

[4] Boulant, J.A. (2006) Journal of Applied Physiology, 100, 1347-1354. https://doi.org/10.1152/japplphysiol.01064.2005

[5] Chan, L.T., Elizabeth, K.C., David, E.L., Yen, E.C.L., Gwendolyn, E.D., Christopher, A.Z. and Zachary, A.K. (2015) Cell, 167, 47-59.

[6] Buonomano, D.V. (2007) Nature Chemical Biology, 3, 594-597. https://doi.org/10.1038/nchembio1007-594

[7] Prigogine, I. (1980) From Being to Becoming. W. H. Freeman and Company, New York, 123.

[8] Erik, P., Mestl, T. and Omholt, S.W. (1995) Journal of Biological Systems, 3, 409-413.

[9] Tansey, E.A. and Johnson, C.D. (2015) Advances in Physiology Education, 39, 139-148. https://doi.org/10.1152/advan.00126.2014 